\title{
Intersection Group Dynamic Subdivision and Coordination at Intraregional Boundaries in Sudden Disaster
}

\author{
Ciyun Lin, ${ }^{1,2}$ Bowen Gong, ${ }^{1,2}$ Zhaosheng Yang, ${ }^{1,2}$ and Xin $Q \mathbf{u}^{2}$ \\ ${ }^{1}$ State Key Laboratory of Automobile Dynamic Simulation, Jilin University, Changchun 130022, China \\ ${ }^{2}$ College of Transportation, Jilin University, Changchun 130022, China \\ Correspondence should be addressed to Bowen Gong; gongbowen@jlu.edu.cn
}

Received 6 June 2014; Accepted 17 August 2014

Academic Editor: Huimin Niu

Copyright (c) 2015 Ciyun Lin et al. This is an open access article distributed under the Creative Commons Attribution License, which permits unrestricted use, distribution, and reproduction in any medium, provided the original work is properly cited.

\begin{abstract}
This paper aims at the traffic flow agglomeration effect characteristics and rapid evacuation requirement in sudden disaster; operation time of intraregional boundaries traffic signal coordination was presented firstly. Then intraregional boundaries intersection group dynamic subdivision and consolidation method based on relative similarity degree and similarity coefficient of adjacent intersections was put forward. As to make the traffic control strategy adapt to traffic condition of different intraregional boundaries intersection groups, this paper proposes an intraregional boundaries traffic signal coordination and optimization technology based on organic computing theory. Finally, this paper uses Delphi 7.0, MapX, and Oracle developing a software package, combined with Paramics V6 Simulator to validate the methods of this paper. The result shows that it can obviously improve disaster affected regional traffic signal control efficiency which reduces average traffic delay by $30-35 \%$, decreases vehicle queue by more than $20 \%$ and reduces evacuation time more than $13.06 \%$.
\end{abstract}

\section{Introduction}

In urban traffic signal control system, any kind of traffic signal control strategy is established in given road geometry conditions and traffic flow characteristics. Only when the traffic network road geometry and its traffic flow characteristics meet or are close to application conditions of traffic signal control strategy, traffic signal control system operation efficiency and effect can be in the best status. Therefore, in accordance with different traffic flow characteristics and performing the suitable traffic signal control strategy, experienced traffic engineer uses the method of dynamical subdivision intersection form intersection groups or consolidate intersection to intersection groups based on traffic flow spatial-temporal distribution characteristics and traffic flow platoon dispersion characteristics.

For the past few years, as the urban traffic congestion is increasing seriously, the requirement of enhancing traffic signal control efficiency and effect is becoming the first and foremost. The method to dynamically subdivide and consolidate intersection groups plays an important role in improving traffic signal control efficiency and adapting to the changing traffic flow in urban network. Moreover, it has become a hot topic which is widely researched in the last years around the world. Lin and Tsao [1] studied the intersection group dynamical subdivision and consolidation in regional traffic signal control system based on searching algorithm in order to conform to the different traffic demands of time of day. Moore and Jovanis [2] using traffic signal cycle, traffic flow, and length of adjacent intersection as the division and consolidation principle, with the data provided by traffic flow guidance system, proposed a dynamical subdivision method for regional traffic signal control system. Wong [3] presented an approach using the parameters of cycle ratio, offset ratio, and split ratio to decide whether to divide from intersection group or consolidate to intersection group. Pranevicius and Kraujalis [4], using fuzzy control theory, put forward an intersection group fuzzy and dynamical subdivision and consolidation method based on coordinated coefficients. Based on the traffic network hypergraph division model, Chandler [5] raised a high-speed intersection group subdivision and consolidation method for traffic control 
network to solve the large-scale traffic signal control system. Wang and Bie [6] put forward an intersection group subdivision model for coordination of traffic signal control system using correlation degree of adjacent intersections. Hsu and Peeta [7], according to the correlation of adjacent intersections on the basis of spectral analysis, proposed three adaptive subdivision and consolidation methods for regional traffic signal control system. Lee et al. [8], using the genetic algorithm and dimension reduction processing to optimize the traffic signal control strategy for intersection group in regional traffic signal control system, presented a set of subdivision and consolidation methods for intersection group to traffic signal control and coordination.

However, the methods of intersection group subdivision and consolidation are mainly focused on the interior of regional traffic signal control system. And according to the traffic flow status in adjacent intersections, these papers deciding whether to subdivide from the intersection group or consolidate to intersection group are under the normal traffic network and traffic flow conditions. Furthermore, current traffic signal control systems with the regional or intersection group as its units operate independently; they are unable to subdivide or consolidate between regional boundaries intersections to form an individual intersection group and lack signal coordination between regional boundaries intersections. Therefore, traffic blocking often occurs in the road sections of intraregional boundaries, which can quickly lead to traffic congestion at interregional boundaries, even more to the all traffic signal control network. In sudden disaster especially, a large number of people and logistics in disaster affected area need imminent evacuation to nearby emergency evacuation point in the adjacent region. But with the current regional traffic signal control mode, the adjacent regional traffic signal control system unable to foresee the large number of traffic flows is coming at the entrance road section in the intraregional boundaries and is unable to adjust the traffic signal control strategy real-time to let the traffic flow pass through the boundaries intersection quickly. So traffic congestion is likely to occur in the intraregional boundaries, which may directly affect the emergency rescue work.

As to solve the problem mentioned above, this paper aims at the traffic flow agglomeration effect characteristics and rapid evacuation requirement in sudden disaster and attempts to use intraregional boundaries intersection group subdivision and consolidation, intraregional boundaries intersection group traffic signal control strategy optimization, and intraregional boundaries intersection groups coordination to divert and rapidly dissipate the traffic flow in intraregional boundaries, preventing traffic congestion occurring at the intraregional boundaries, in order to let emergency rescue work be implemented faster and smoother in sudden disaster.

\section{Deciding the Operation Time of Coordination}

The objective of deciding the operation time of traffic signal coordination between intraregional boundaries intersection groups is to make the intraregional boundaries intersection group running with suitable traffic signal control strategy under different traffic flow conditions so that it can improve the traffic capacity of intraregional boundaries intersection group. In this paper, we use the interconnected index of adjacent intersections in intraregional boundaries as the decision and evaluation index of operation time of traffic signal coordination for intraregional boundaries [9]:

$$
I=0.5 \cdot \frac{((\chi \cdot q / Q)-1)}{(1+T)},
$$

where $I$ is interconnected index; $T$ is average travel time between adjacent intersections in intraregional boundaries; $\chi$ is the lane numbers that traffic flow can enter into downstream intersection; $q$ is the traffic volume of straight lanes in upstream intersection; $Q$ is the total traffic volume from upstream intersection. Consider

$$
\begin{gathered}
T=\frac{L}{V}, \\
Q=q+k q=(1+k) q,
\end{gathered}
$$

where $L$ is length of adjacent intersection in intraregional boundaries; $V$ is average travel speed; and $k$ is the sum of left turn ratio and right turn ratio.

Put (2) into (1):

$$
I=0.5 \cdot \frac{((\chi /(1+k))-1)}{(1+L / V)} .
$$

When $I>\delta_{1}$ or $I<\delta_{2}$, it is the time to subdivide intersection from intersection group or consolidate intersection to intersection group and formulate different traffic signal control intersection group in intraregional boundaries, where $\delta_{1}$ is the threshold limit value of subdivision and $\delta_{2}$ is the threshold limit value of consolidation.

\section{Intersection Group Dynamical Subdivision or Consolidation}

3.1. Impact Factor Analysis. Traffic signal control system is made up of signalized intersections in traffic network. Each signalized intersection has its status variables and control variables. Status variables describe intersection's geometric design and traffic flow at each inbound link. Control variables describe traffic control parameters, consisting of cycle, offset, and split and the capacity at specified performance levels. These variables are interrelated and interacting. At the same time, traffic flow in adjacent intersections has the potential incidence relation of similarity and coupling. With the passage of time and the transition of space, relationships between adjacent intersections are changing continuously. In sudden disaster, besides the changing relationships between adjacent intersections, the layout and the function of the traffic network are also changing by road damage, road closure, separation by traffic management, and so on. Therefore, in sudden disaster, it needs more functions to make the traffic signal control strategy to suit for the changing traffic flow and traffic network and let each intersection group in regional 
traffic signal control system have its own control objectives based on its traffic flow characteristics. The intersection group subdivision and consolidation are considered to be the best way to achieve the goal as mentioned above. It can improve the practicability, reliability, and instantaneity of traffic signal control models and algorithms in regional traffic signal control system.

According to the traffic flow characteristics and traffic network geometric topology information, the data attributes of signalized intersection can be described in different forms. From the perspective of time factors, status variables and control variables can be classified as dynamic variables and static variables. From the perspective of space factors, status variables and control variables can be classified as link variables and node variables. In this paper, $X$ stands for the state of the signalized intersection. Consider

$$
\begin{gathered}
X_{j}^{i}=e_{i, j}^{\text {node }}+e_{i, j}^{\text {link }}, \\
e_{i, j}^{\text {node }}=\left[A_{i, j}^{\text {node,static }}, A_{i, j}^{\text {node,dynamic }}\right], \\
e_{i, j}^{\text {link }}=\left[A_{i, j}^{\text {link,static }}, A_{i, j}^{\text {link,dynamic }}\right],
\end{gathered}
$$

where $X_{j}^{i}$ is the state of $j$ th intersection in $i$ th intersection group. $e_{i, j}^{\text {node }}$ is the node matrix of $j$ th intersection in $i$ th intersection group. $e_{i, j}^{\text {link }}$ is the link matrix of $j$ th intersection in $i$ th subzone intersection group. Node and link matrices both include static and dynamic vectors that describe node or link's status variable and control variable. $A_{i, j}^{\text {node,static }}=\left[T_{i, j}, K_{i, j}\right.$, $\left.P_{i, j}\right], T, K, P$ mean intersection geometric type, critical intersection or not, and signal phase number, respectively. $A_{i, j}^{\text {node,dynamic }}=\left[\mathrm{Ct}_{i, j}, \mathrm{Sat}_{i, j}, \mathrm{Ca}_{i, j}\right], \mathrm{Ct}$, Sat, Ca mean signal cycle, saturation, and capacity of the intersection, respectively. $A_{i, j}^{\text {link,static }}=\left[\mathrm{Lk}_{i, j}, \mathrm{Id}_{i, j}, \mathrm{Lg}_{i, j}, \mathrm{Ld}_{i, j}\right], \mathrm{Lk}, \mathrm{Id}, \mathrm{Lg}, \mathrm{Ld}$ mean the vector of each inbound link has a connected intersection or not, the connected intersection id, the length of the link and lane numbers in the link, respectively. $A_{i, j}^{\text {link,dynamic }}=\left[V_{i, j}\right.$, $\left.v_{i, j}, O_{i, j}, \lambda_{i, j}\right], V, v, O, \lambda$ mean the vector of each inbound link's volume, average speed, average occupancy, and split of signal, respectively.

3.2. Standardization State Matrix. Both signalized intersection's status variables and control variables make up the intraregional boundaries traffic signal control network's status. The status of intraregional boundaries traffic signal control network can be described as the status matrix $S[10]$ :

$$
S=\left[\begin{array}{cccc}
A_{0,1}^{\text {nodestatic }} & A_{0,1}^{\text {node,dynamic }} & A_{0,1}^{\text {linkstatic }} & A_{0,1}^{\text {link,dynamic }} \\
A_{0,2}^{\text {node,static }} & A_{0,2}^{\text {node,dynamic }} & A_{0,2}^{\text {link,static }} & A_{0,2}^{\text {link,dynamic }} \\
\vdots & \cdots & & \\
A_{0, n}^{\text {node,static }} & A_{0, n}^{\text {node,dynamic }} & A_{0, n}^{\text {linkstatic }} & A_{0, n}^{\text {link,dynamic }}
\end{array}\right] .
$$

In order to eliminate difference among status variables and control variables, it is needed to transform the matrix $S$ to standardization matrix. In intraregional boundaries traffic signal control network status matrix $S$, towards the status variables, let

$$
r_{i j}=\frac{\left(y_{i j}-y_{i}^{\min }\right)}{\left(y_{i}^{\max }-y_{i}^{\min }\right)},
$$

where $Y \in$ status variables and $i=(1,2, \ldots, n)$.

For control variables, let

$$
r_{i j}=\frac{\left(y_{i}^{\max }-y_{i j}\right)}{\left(y_{i}^{\max }-y_{i}^{\min }\right)},
$$

where $Y \in$ control variables and $i=(1,2, \ldots, n)$.

So the standardization matrix $R$ is

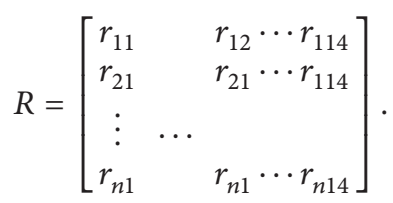

Traffic network layout and its functional design, traffic flow distribution, and characteristics decide the intersection in the network that will undertake different objectives and make different influence on traffic flow operating. The significant degree of intersection in the network not only is related to the geographic position and geometric topology in the network, but also has a close relationship with the dynamic traffic flow that passes through or comes to the intersection. However, the role of influence factors that impact intersection is different. And the value of influence is difficult to define as the diversity, complexity of traffic environment, and limited awareness of human acknowledge. So, in this paper, we use the theory of multiple attribute decision making (MADM) to measure the intersection status matrix of intraregional boundaries traffic signal control network [11]. Consider

$$
\begin{gathered}
W=\left(w_{1}, w_{2}, \ldots, w_{14}\right), \\
Z=\left[\begin{array}{cccc}
w_{1} r_{11} & w_{2} r_{12} & \cdots & w_{14} r_{114} \\
w_{1} r_{21} & w_{2} r_{22} & \cdots & w_{14} r_{214} \\
\vdots & & & \\
w_{1} r_{n 1} & w_{2} r_{n 2} & \cdots & w_{14} r_{n 14}
\end{array}\right]=\left(z_{i j}\right)_{n \times 14},
\end{gathered}
$$

where $W$ is the weight of variables. $Z$ is the standardization matrix of intraregional boundaries traffic signal control network.

3.3. Hamming Osculating. Define the ideal state as a signalized intersection in the best status that can be subdivided from an intersection group in intraregional boundaries and improve the traffic signal control efficiency. And the negative state is a signalized intersection in the best status that can be consolidated to an intersection group in intraregional boundaries or else can reduce traffic signal control performance. The ideal state is expressed as $A^{+}$:

$$
A^{+}=\left\{A_{1}^{+}, A_{2}^{+}, A_{3}^{+}, \ldots, A_{14}^{+}\right\} .
$$


The negative state is expressed as $A^{-}$:

$$
A^{-}=\left\{A_{1}^{-}, A_{2}^{-}, A_{3}^{-}, \ldots, A_{14}^{-}\right\}
$$

Then, the distance of current status of signalized intersection $X_{j}^{i}$ to the ideal state that should be subdivided from an intersection group in intraregional boundaries can be expressed as

$$
\mu_{i}^{+}=\sqrt{\sum_{j=1}^{14}\left(z_{i j}-A_{j}^{+}\right)^{2}}
$$

The distance of current status of signalized intersection $X_{j}^{i}$ to the negative state that would be consolidated to an intersection group in intraregional boundaries can be expressed as

$$
\mu_{i}^{-}=\sqrt{\sum_{j=1}^{14}\left(z_{i j}-A_{j}^{-}\right)^{2}}
$$

Signalized intersection $X_{j}^{i}$ is suitable to be subdivided from an intersection group or to be consolidated into an intersection group in intraregional boundaries which is decided by hamming osculating. Consider

$$
\omega_{i}=\frac{\mu_{i}^{-}}{\left(\mu_{i}^{+}+\mu_{i}^{-}\right)}
$$

where $0<\omega_{i}<1$. As $\omega_{i}$ is close to $0, \mu_{i}^{-}$approaches 0 ; traffic signal control performance reduces when the signalized intersection consolidates to an intersection group or subdivides from an intersection group in intraregional boundaries. And as $\omega_{i}$ approaches 1 and $\mu_{i}^{+}$approaches 0 , traffic signal control system will get better performance when consolidating a signalized intersection to an intersection group or subdividing it from an intersection group in intraregional boundaries. And the hamming osculating of the whole intraregional boundaries traffic network is

$$
\omega=\left[\omega_{1}, \omega_{2}, \ldots, \omega_{14}\right] .
$$

3.4. Coefficient of Adjacent Intersection. In algebra, a cosine is usually used between two angles to represent the similarity between vectors; in this paper, we show the similarity of two signalized intersections by calculating the cosine between two state vectors of adjacent signalized intersections:

$$
\cos \theta=\frac{|x, y|}{|x|} \cdot|y|
$$

So the similarity matrix of intraregional boundaries signalized intersections is

$$
\operatorname{coef}=\left[\begin{array}{cccc}
1 & \cos \theta_{12} & \cdots & \operatorname{con} \theta_{1 n} \\
\cos \theta_{21} & 1 & \cdots & \cos \theta_{2 n} \\
\vdots & & \cdots & \\
\cos \theta_{n 1} & \cos \theta_{n-1 * n} & \cdots & 1
\end{array}\right] .
$$

3.5. Dynamic Cluster Analysis. Dynamic cluster analysis is used to analyze hamming osculating and coefficient of adjacent intersections in intraregional boundaries. The processing is described as below.

Step 1. Make $m$ cluster and set initial cluster center in a rough set for hamming osculating and coefficient of adjacent intersections, respectively.

(1) Calculate the distance between intersections to its adjacent intersection.

(2) Order the distance from small to large.

(3) The similar distance elements are classified as a class, initializing the $\mathrm{m}$ clusters, and calculate the average value of all objects corresponding coordinates in each cluster, as the initial cluster centers.

Step 2. Based on the cluster radius and interval, sort out the hamming osculating $\omega$ and coefficient $\cos \theta$, respectively.

Step 3. When the signalized intersection's hamming osculating $\omega$ and coefficient $\cos \theta$ are classified into the same cluster, consolidate the signalized intersection to an intersection group and classify it to the intersection group cluster.

Step 4. When all the signalized intersections have been classified, calculate the distance between the clusters and output and sort the distance of adjacent intersections.

(1) Calculate link segment saturation as

$$
\psi_{a}(t)=\frac{u_{a}(t)}{S_{a}}
$$

where $\psi_{a}(t)$ is the saturation of the link segment. $u_{a}(t)$ is the flow rate of the link $a$ at time $t . S_{a}$ is the saturation in the link segment $a$.

(2) Assume that there are $n$ link segments in the intersection group, boundaries intersections contain $m$ link segments ( $m \leq n$ ); the threshold saturation of the intersection group that decides to start cluster analysis for subdividing and consolidating is

$$
K_{1}(t)=\sqrt{\frac{\psi_{1}^{2}(t)+\psi_{2}^{2}(t)+\cdots+\psi_{m}^{2}(t)}{m}} .
$$

The saturation of remaining link segments is

$$
K_{2}(t)=\sqrt{\frac{\psi_{1}^{2}(t)+\psi_{2}^{2}(t)+\cdots+\psi_{n-m}^{2}(t)}{n-m}} .
$$

(3) The saturation of intersection group can be calculated by weighting coefficient that considers the importance of the link:

$$
K(t)=\rho K_{1}(t)+(1-\rho) K_{2}(t)
$$

Step 5. Confirm the threshold value $\zeta$ based on cluster distance. 


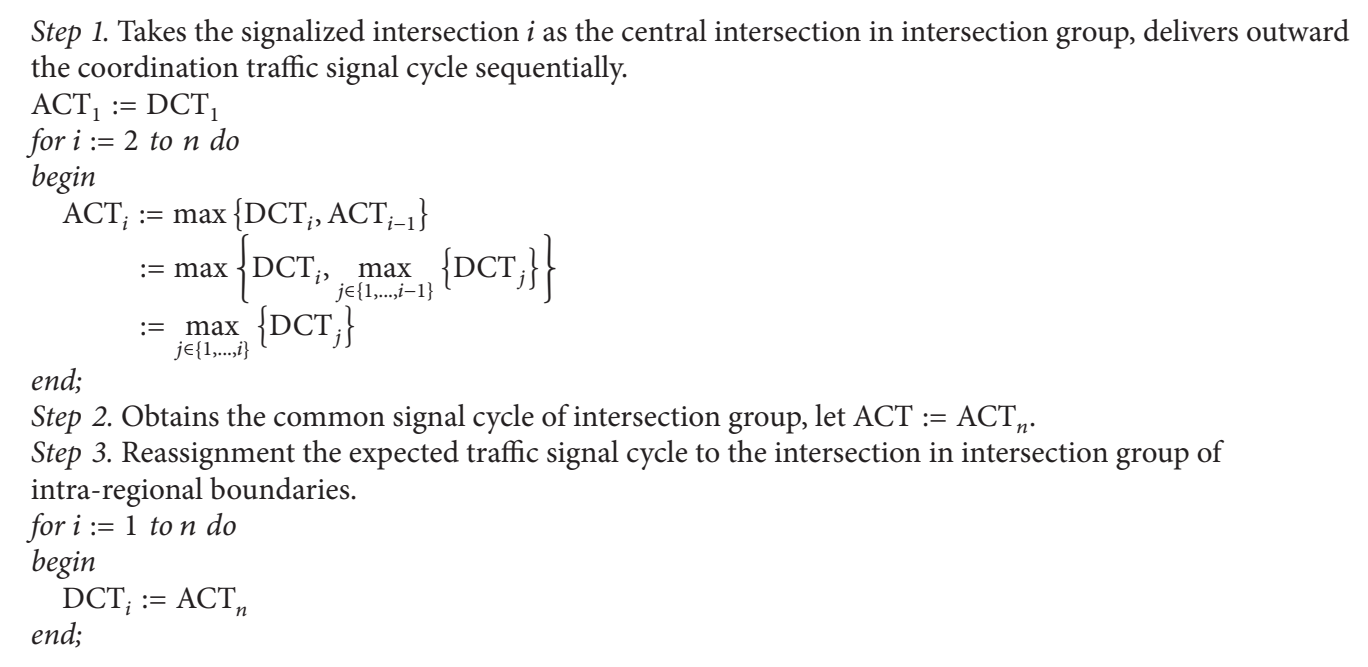

Algorithm 1

Step 6. Merge the cluster when the cluster distance is less than the threshold value $\zeta$ and then export the cluster result of the intraregional boundaries.

Step 7. Determine whether clustering results are rational or not and finish the cluster analysis and output the cluster results if the answer is yes; then wait for the next cycle. If it returns NO, go back to Step 2 .

\section{Traffic Signal Optimization and Coordination for Intraregional Boundaries}

Organic computing is a form of distributed and biologically inspired computing with organic properties. It develops from the idea that central nervous system of human neural network maintains and automatically adjust the balance of body system based on the exogenous and endogenous environment information perceived by human organics. Organic computing has emerged recently as a challenging vision for future information processing system. It has outstanding performance in the distributed, dynamic and heterogeneous network environment. Organic computing system is a technical system which is equipped with sensors and actuators as to be aware of the environment accurately, communicate freely, and organize the response plan automatic like organisms. Organic computing system adapts dynamically to exogenous and endogenous changes, solves the conflict of different systems, and responds to the unpredictable emergency problems by its functional characteristics of self-properties, such as selfmonitoring, self-organization, and self-optimization [12]. This paper uses organic computing system techniques to automatically detect and monitor the traffic flow in disaster affected region and optimize the traffic signal to resolve the conflict problems in intraregional boundaries when emergency evacuation occurs in sudden disaster. Organic computing for intraregional boundaries traffic signal control system is composed by four modules. There are traffic flow selfmonitoring, traffic signal self-optimization, traffic signal coordination knowledge self-organization, and traffic signal control plan self-classification.

4.1. Traffic Flow Self-Monitoring. Traffic flow self-monitoring module is the sensors and actuators of the organic computing system, which was used to percept the environment condition and implement the control commands. In intraregional traffic signal control system, traffic flow self-monitoring uses traffic detectors (including loop coil vehicle detector, microwave vehicle detector, and video detector) which distribute in traffic network to detect and monitor the traffic flow condition in intraregional boundaries and provide reliable, real-time, and comprehensive traffic information for traffic signal control system.

\subsection{Traffic Signal Self-Optimization}

4.2.1. Traffic Signal Cycle Online Adjustment. Define the $\mathrm{DCT}_{i}$ as the expected traffic signal cycle of signalized intersection $i$ in intraregional boundaries. $\mathrm{OCT}_{i}$ is the optimal traffic signal cycle calculated by Webster cycle formula based on real-time traffic flow data. $\mathrm{DCT}_{i}=\mathrm{OCT}_{i}$ or $\mathrm{DCT}_{i}=$ $(1 / 2) \mathrm{OCT}_{i} \cdot n, n \in[1,2, \ldots, n]$. Define the $\mathrm{ACT}_{i}$ as the coordination traffic signal cycle of signalized intersection $i$ in intraregional boundaries. It is the signal cycle that local traffic signal controllers agree to coordinate and optimize. The common signal cycle of intersection group in intraregional boundaries is calculated as in Algorithm 1 [13].

Thus, the signal intersection of intersection group in intraregional boundaries can optimize and dynamically adjust the traffic signal based on real-time traffic flow data.

4.2.2. Traffic Signal Split Optimization. Traffic signal split optimization is to make sure that all the inbound traffic flows can 
go through the signalized intersection at the current traffic signal cycle. The mathematical expression is

$$
\begin{aligned}
\tilde{q}_{i j}^{\text {in }}(k) & -\widetilde{v}_{i j}^{\text {exit }}(k)+\widetilde{v}_{i j}^{\text {demand }}(k)-\widetilde{q}_{i j}^{\text {out }}(k) \\
= & \left(1-r_{i j}^{0}\right) \widetilde{q}_{i j}^{\text {in }}(k)+\widetilde{v}_{i j}^{\text {demand }}(k)-\widetilde{q}_{i j}^{\text {out }}(k)=0 .
\end{aligned}
$$

The dynamic kinematic equation of traffic flow between adjacent intersections in intraregional boundaries is

$$
\begin{aligned}
Q_{i j}(k+1)= & Q_{i j}(k)+C_{j}(k) \\
\times & {\left[\left(1-r_{i j}^{0}\right) \sum_{w \in I L_{i}} \frac{r_{w j}^{i} \cdot S_{w i} \cdot \sum_{p \in \mathrm{ROW}_{w i}} g_{w i}^{p}(k)}{C_{i}(k)}\right.} \\
& \left.+v_{i j}^{\text {demand }}(k)-\frac{S_{i j} \cdot \sum_{p \in \mathrm{ROW}_{i j}} g_{i j}^{p}(k)}{C_{j}(k)}\right] .
\end{aligned}
$$

Let $C_{j}(k)=C_{i}(k)=C$ and put (22) into (23); the dynamic kinematic equation can be rewritten as

$$
\begin{aligned}
Q_{i j}(k+1)= & Q_{i j}(k)+C_{j}(k) \\
\times & {\left[\left(1-r_{i j}^{0}\right) \sum_{w \in I L_{i}} \frac{r_{w j}^{i} \cdot S_{w i} \cdot \sum_{p \in \mathrm{ROW}_{w i}} \Delta g_{w i}^{p}(k)}{C}\right.} \\
& \left.+\Delta v_{i j}^{\text {demand }}(k)-\frac{S_{i j} \cdot \sum_{p \in \mathrm{ROW}_{i j}} \Delta g_{i j}^{p}(k)}{C}\right],
\end{aligned}
$$

where $Q_{i j}(k)$ is the traffic flow volume in link segment $L_{i j}$ between adjacent intersections $N_{i}$ and $N_{j}$ at the time $k$. $q_{i j}^{\text {in }}, q_{i j}^{\text {out }}$ are the traffic flow rate income and depart the link segment $L_{i j}$, respectively. $v_{i j}^{\text {exit }}, v_{i j}^{\text {demand }}$ are the traffic flow rate of trip attraction and trip generation by link segment $L_{i j}$, respectively. $C_{j}(k)$ is the traffic signal cycle length of intersection $N_{j}$ at the $k$ th time. Consider

$$
\begin{gathered}
\Delta g_{i j}^{p}(k)=g_{i j}^{p}-\widetilde{g}_{i j}^{p}, \\
\Delta v_{i j}^{\text {demand }}=v_{i j}^{\text {demand }}-\widetilde{v}_{i j}^{\text {demand }} .
\end{gathered}
$$

And the state equation of traffic signal split optimization for intersection $N_{j}$ can be described as

$$
x(k+1)=x(k)+A \Delta g(k)+B \Delta v^{\text {demand }}(k) .
$$

Assume that the change of link segment inner traffic flow generation can be ignored, and let $\Delta v^{\text {demand }}(k)=0$; (26) can form a linear quadratic optimal control problem:

$$
x(k+1)=x(k)+A \Delta g(k) .
$$

The performance index of the linear quadratic optimal control problem is

$$
\mathfrak{J}=\frac{1}{2} \sum_{k=0}^{\infty}\|x(k)\|_{Q}^{2}+\|\Delta g(k)\|_{R}^{2} .
$$

Then calculus of variations is used to solve the traffic signal feedback control law of the linear quadratic optimal control problem [14]:

$$
g(k)=g(k-1)-\alpha Q(k)-\beta Q(k-1),
$$

where $\alpha, \beta$ are the control coefficients.

4.2.3. Traffic Signal Offset Synchronization. Consider the intersection group as an intersection unit $I$, and let $o_{i-1}$ be the traffic signal offset of intersection groups $i-1 ; p_{i-1}$ is the coordination direction of intersection groups $i-1, d_{i-1, i}$ is the time that vehicle form intersection groups $i-1$ to intersection groups $i, q_{i}$ is the dissipation time of traffic flow waiting at intersection groups $i$ in intraregional boundaries, $r_{i}$ is the remaining current traffic signal time of intersection groups $i, T_{i}$ is the current traffic signal cycle length of intersection groups $i, s_{i}$ is the traffic signal cycle start time of intersection groups $i$, and $t_{i}$ is the next traffic signal cycle execution time of intersection groups $i$.

The equations of the traffic signal offset are as follows:

$$
\begin{gathered}
o_{i}=\left(o_{i-1}+p_{i-1}+d_{i-1, i}-p_{i}-q_{i}\right) \bmod \text { ACT } \\
t_{i}=s_{i}+o_{i}-r_{i}-T_{i} .
\end{gathered}
$$

If $t_{i}<C_{\min }$, then $t_{i}:=t_{i}+$ ACT.

In intersection group, make the key signalized intersection as the standard point, and $o_{i, j}$ is the traffic signal offset between intersection $N_{i}$ and $N_{j}$. Then the execution traffic signal cycle time of the other intersections in intersection group is

$$
t_{i, j}=s_{i, j}+o_{i, j}-r_{i, j}-T_{i, j}
$$

4.3. Traffic Signal Coordination Knowledge Self-Organization. In advanced traffic signal control system, the knowledge discovery rules for optimizing traffic signal timing are based on traffic flow environment. When traffic flow status and conditions are changing, the quality of the knowledge discovery rule is needed to reevaluate firstly. And then traffic signal coordination knowledge self-organization module uses genetic algorithms to classify the traffic flow status and condition based on knowledge structure database. Finally, traffic signal coordination knowledge self-organization module forms a new knowledge discovery rule to suit for the changing traffic flow as to obtain the optimization traffic signal control parameters [15]. This paper uses profit-sharing plan to evaluate the knowledge discovery rule when traffic flow status and condition change. Profit-sharing plan (PSP) provides complementary information utility for each rule when forming new rule as to adapt the traffic flow environment.

Assume that the strength of PSP to modify the activity rule $R_{i}$ at $t$ time is $S_{i}(t)$; then

$$
\begin{gathered}
S_{i}(t+1)=S_{i}(t)-b S_{i}(t)+b p(t), \\
S_{i}(t)=(1-b)^{t} S_{i}(0)+b \sum_{i=1}^{t}(1-b)^{t-i} p(i-1),
\end{gathered}
$$




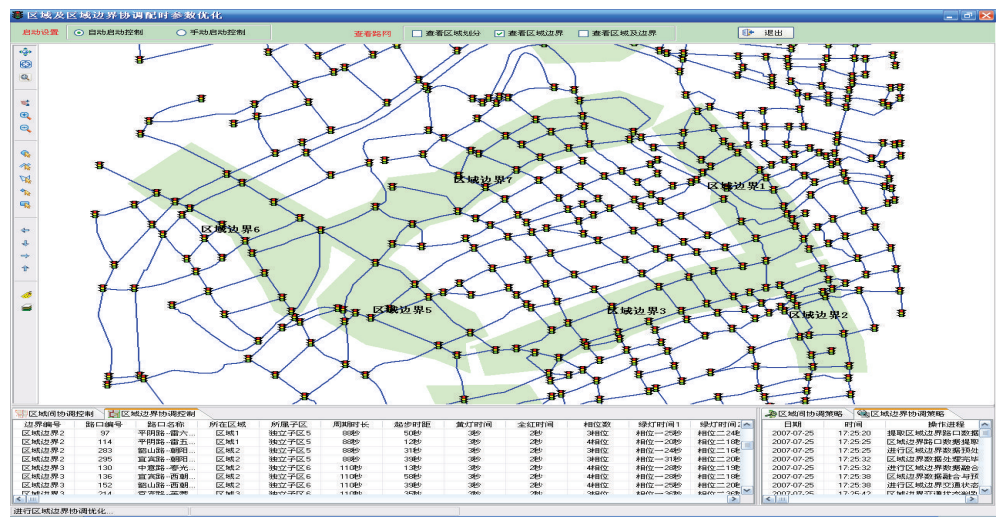

FIGURE 1: Snapshot of simulation network from the GUI of intraregional traffic signal coordinated software package.

where $p(t)$ is the external reward at the end of the knowledge discovery rule updating. $(1-b)$ is the index attenuation factor. If $b$ is small enough, then $S_{i}(t)$ has the mean value of $p(t)$.

Let the value of external reward $p(t)$ be a constant value $p^{*}$; then $S_{i}(t)$ will converge to an equilibrium value $S_{i}^{*}$ :

$$
S_{i}^{*}=\lim _{t \rightarrow \infty}\left[(1-b)^{t} S_{i}(0)+b \sum_{i=1}^{t}(1-b)^{t-i} p^{*}\right]=p^{*} .
$$

With the constant value $p^{*}$, PSP will reduce the error as in the following rate:

$$
\begin{gathered}
E_{i}(t)=p^{*}-S_{i}(t), \\
\Delta E_{i}=E_{i}(t+1)-E_{i}(t) \\
=S_{i}(t)-S_{i}(t+1) \\
=-b\left(p^{*}-S_{i}(t)\right) \\
=-b E_{i}(t) .
\end{gathered}
$$

4.4. Traffic Signal Control Plan Self-Classification. Classification learning aims at searching for the most suitable traffic signal control parameters for the intersection in intraregional boundaries under the current traffic flow status and condition. The knowledge base system and online parameter optimization system offer the suitable traffic signal control strategy, so that traffic signal control system operation efficiency and effect can be in the best status. That can improve the emergency evacuation speed in sudden disaster. The classification learning processing is as follows [15].

Step 1. Insert all the current intersection traffic flow information and traffic signal timing parameters data into the message table.

Step 2. Compare all record data in the message table with classifying conditions in all classifiers and extract the traffic flow status and the corresponding signal timing parameters from all matching records.

Step 3. Send traffic signal timing parameters optimization by microscopic simulation software to the new message table for the traffic flow status cannot find the matching traffic signal timing parameter.

Step 4. Use new message table to replace the old messages table and add the extracted matching record to the new message table.

Step 5. Translate messages in the message table into the requirement of output interface, providing traffic signal control strategy and timing parameters for current traffic flow.

Step 6. Return to Step 1.

\section{Simulation and Verification}

A part of Shanghai CBD traffic network was selected to simulate the intraregional boundaries intersection group subdivision and consolidation for traffic signal control and coordination. The traffic network is composed of 422 intersections and 2881 directional links. As to verify the methods proposed by this paper, the traffic network is divided into five traffic signal control regions, and the five regions traffic signal control systems form eight intraregional boundaries. The simulation traffic network is shown as in Figure 1.

Simulation input data come from loop coils vehicle detectors and video cameras vehicle detectors in July 14 to 27, 2007. The interval of data collection is 5 minutes. These detectors collect traffic parameters including traffic flow volume, speed, and occupancy rate. As to simulate the influence on traffic network in sudden disaster, this paper uses random disturbance factor to randomly reduce road capacity of the traffic network [16]. And this paper defines the threshold value that an intersection needs to be subdivided from the intraregional boundaries intersection group as $\delta_{1}=0.45$. And the threshold value that an intersection should be consolidated into the intraregional boundaries intersection group is $\delta_{2}=0.75$. The verification work was done as the following procedure.

Step 1. Input the traffic network and traffic flow data into Synchro V6 signal optimization software and obtain traffic 
TABLE 1: Traffic flow performance in intraregional boundaries under different traffic signal control strategies.

\begin{tabular}{lccccccccc}
\hline \multirow{2}{*}{ Traffic flow status } & \multirow{2}{*}{ Statistics item } & \multicolumn{2}{c}{ Delay } & \multicolumn{2}{c}{ Speed } & \multicolumn{2}{c}{ Saturation } & \multicolumn{2}{c}{ Queen } \\
& & Mean value & STDEV & Mean value & STDEV & Mean value & STDEV & Mean value & STDEV \\
\hline \multirow{4}{*}{ Free } & SP & 4.227 & 1.306 & 48.13 & 5.565 & 0.5197 & 0.1529 & 13.22 & 5.714 \\
& RP & 4.212 & 1.263 & 48.24 & 5.397 & 0.5233 & 0.1557 & 12.13 & 5.512 \\
& BP & 4.165 & 1.266 & 48.56 & 5.474 & 0.5132 & 0.1537 & 10.81 & 4.273 \\
& REDEV I\% & -1.467 & -3.063 & 0.8934 & -1.635 & -1.251 & 0.5232 & -18.23 & -25.22 \\
& REDEV II\% & -1.116 & 0.2375 & 0.6633 & 1.427 & -1.930 & -1.285 & -10.88 & -22.48 \\
\hline \multirow{5}{*}{ Smooth } & SP & 28.67 & 6.094 & 41.93 & 8.841 & 0.6808 & 0.1721 & 35.69 & 4.37 \\
& RP & 25.43 & 5.873 & 42.17 & 7.064 & 0.6637 & 0.1673 & 34.66 & 3.25 \\
& BP & 16.84 & 3.257 & 43.06 & 5.563 & 0.6551 & 0.1024 & 24.37 & 6.45 \\
& REDEV I\% & -41.26 & -46.55 & 2.695 & -37.08 & -3.775 & -40.50 & -31.72 & 47.60 \\
& REDEV II\% & -33.78 & -44.54 & 2.111 & -21.25 & -1.296 & -38.79 & -29.69 & 98.46 \\
\hline \multirow{6}{*}{ Congestion } & SP & 44.27 & 3.714 & 21.27 & 3.152 & 0.9394 & 0.1271 & 50.58 & 6.991 \\
& RP & 41.32 & 4.257 & 23.61 & 3.231 & 0.9051 & 0.1104 & 47.15 & 9.342 \\
& BP & 30.27 & 3.335 & 28.39 & 4.173 & 0.8609 & 0.0964 & 34.66 & 3.25 \\
& REDEV I\% & -31.62 & -10.20 & 33.47 & 32.39 & -8.356 & -24.15 & -31.47 & -53.51 \\
& REDEV II\% & -26.74 & -21.66 & 20.25 & 29.16 & -4.883 & -12.68 & -26.49 & -65.21 \\
\hline
\end{tabular}

Note. STDEV means standard deviation. REDEV means relative deviation. REDEV I\% $=(\mathrm{BP}-\mathrm{SP}) * 100 / \mathrm{SP}$. REDEV II\% $=(\mathrm{BP}-\mathrm{RP}) * 100 / \mathrm{RP}$.

signal control parameters of every signalized intersection in the traffic network output by Synchro V6.

Step 2. With microscopic simulation software Paramics V6, Paramics V6 simulator inputs traffic flow data using the data obtained from loop coils vehicle detectors and video cameras vehicle detectors. Signal control plan setting in Paramics V6 simulator uses the signal control parameters that output from Synchro V6 by Step 1. Then run the Paramics V6 simulation to get the intraregional boundaries traffic flow performance indices which are defined as SP.

Step 3. Develop the interregional traffic signal strategic coordination and control algorithm in [17]. With the integrated development environment (IDE) and visual component library (VCL) of Delphi 7.0, MapX 4.5, and Oracle 9i, the interregional traffic signal coordination and control software is released, named as URSC. Paramics V6 simulation signal control plans are replaced by the signal control parameters output from URSC. Then run the Paramics V6 simulation to get the intraregional boundaries traffic flow performance indices which are defined as RP.

Step 4. Develop the interregional traffic signal coordination and control method of this paper; with the integrated development environment (IDE) and visual component library (VCL) of Delphi 7.0, MapX 4.5, and Oracle 9i, the intraregional traffic signal coordination and control software is released, named as UBSC. UBSC GUI is shown in Figure 1. Paramics V6 simulation signal control plans are replaced by the signal control parameters output from UBSC. Then run the Paramics V6 simulation to get the intraregional boundaries traffic flow performance indices which are defined as BP.
Step 5. Compare the performance indices of SP, RP, and BP.

Traffic flow performance in intraregional boundaries is analyzed in three different traffic flow statuses. 7:30 to 9:00 and 16:30 to 19:00 are congestion traffic flow statuses. 10:00 to $14: 30$ is smooth traffic flow status. 5:00 to 7:00 and 15:00 to 16:00 are free traffic flow statuses. Then this paper analyzes the traffic flow performance using the index of average vehicle delay (s), average vehicle speed $(\mathrm{km} / \mathrm{h})$, the average saturation, and the average queue length $(\mathrm{m})$. Traffic flow performance in different traffic flow status was shown in Table 1.

Comparing the traffic flow performance data, there are no significant differences existing in intraregional boundaries traffic flow whether it uses the intraregional boundaries coordination signal control under the free traffic flow status or not. However, it can significantly reduce vehicle queuing in the intraregional boundaries intersection. Under smooth and congestion traffic flow statuses, if the system lacks coordination signal control between intraregional boundaries, it can obviously be seen that, from the GUI of Paramics V6 when running simulation, the traffic flows in upstream links and downstream links within intraregional boundaries show the accumulation phenomenon quickly. If using the intraregional boundaries coordination signal control, the system can effectively slow down the accumulated phenomenon. The accumulating traffic flow can go through the intraregional boundaries smoothly. From the simulation results, we can see that if the system uses UBSC and URSC together under congestion traffic flow status, it can reduce $33.78 \%$ vehicle delay and $29.69 \%$ vehicle queue length compared to only used URSC traffic signal control strategy and reduce $41.26 \%$ vehicle delay and $31.72 \%$ vehicle queue length compared to only Synchro V6 optimization. If the system uses UBSC and 


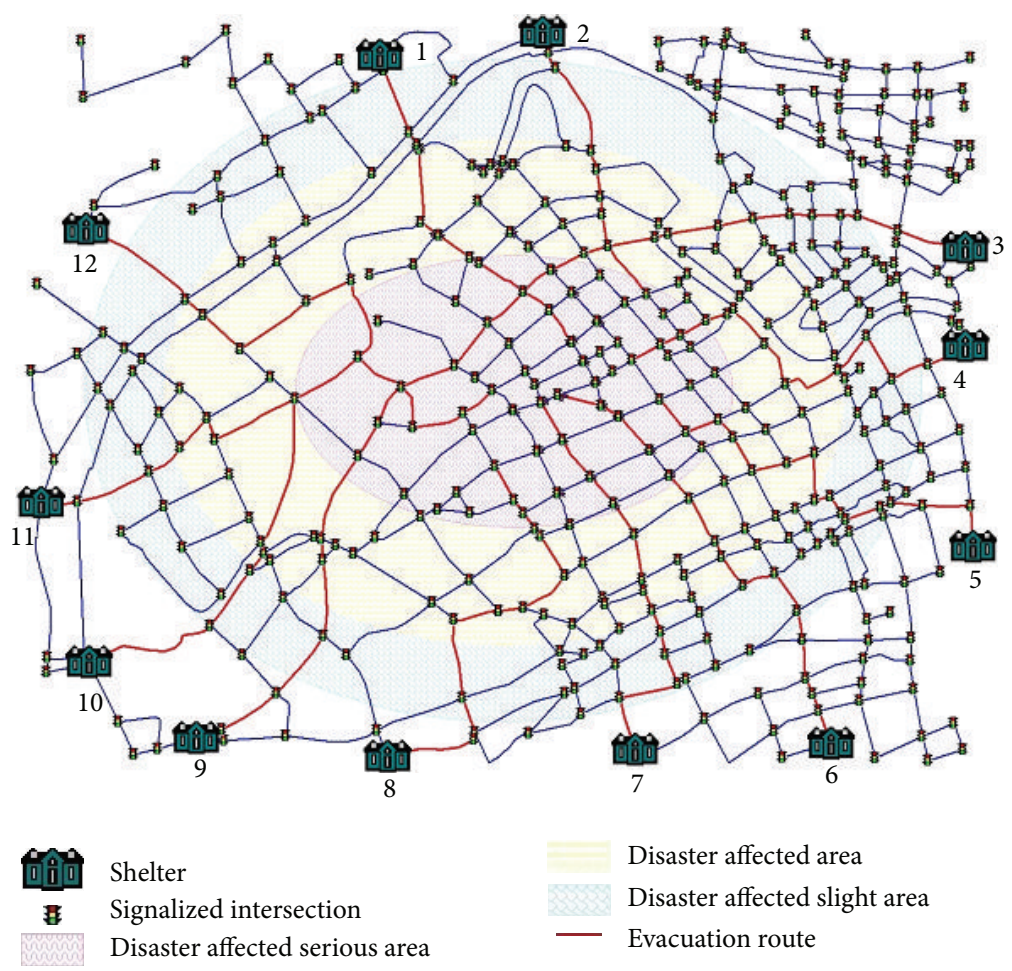

Figure 2: Snapshot of disaster network and evacuation routes.

URSC together under smooth traffic flow status, it can reduce $26.74 \%$ vehicle delay and $26.49 \%$ vehicle queue length compared to only used URSC traffic signal control strategy and reduce $31.62 \%$ vehicle delay and $31.47 \%$ vehicle queue length compared to only Synchro V6 optimization.

Meanwhile, as to verify the emergency evacuation effect of the intraregional boundaries traffic signal control strategy in this paper, this paper optimizes the emergency evacuation routes of the simulation network based on temporal-spatial crowding degree [18] and exports twelve emergency evacuation routes for transport disaster affected people and logistics. Figure 2 shows the disaster condition, emergency evacuation routes, and shelter location.

Simulation was done under the conditions that disaster affected traffic flow volumes are 30,000, 50,000, and 100,000, respectively. Simulator is running with and without the intraregional boundaries traffic signal coordination strategy. Each case runs three times, every time running 7,200 seconds in simulator. Then get the data to analyze the relationship between evacuation time and evacuation volume of traffic flow. The results are shown in Figure 3.

From the simulation results, as the more the traffic flow volume trapped in sudden disaster is, the more the capacity of road in traffic network damage is and the higher the degree of traffic flow burden in traffic network is. The evacuation time is growing with the exponential trend. Under the traffic flow volumes are 30,000, 50,000, and 100,000 in disaster affected region, with intraregional boundaries traffic signal coordination strategy being able to reduce about $13.06 \%, 15.22 \%$, and $20.16 \%$ evacuation time, respectively, compared to without intraregional boundaries traffic signal coordination strategy.

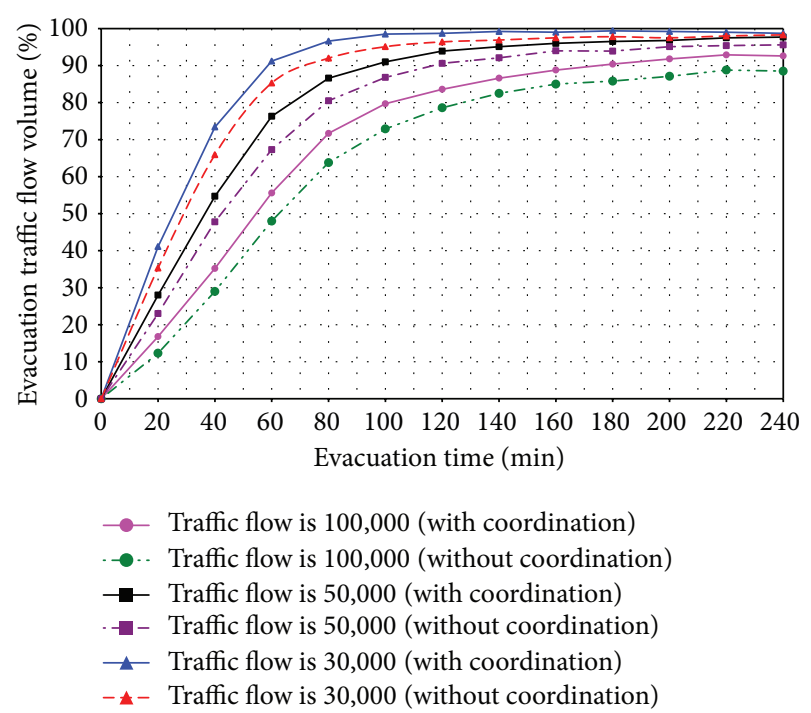

FIGURE 3: The relationship between evacuation time and evacuation volume of traffic flow.

\section{Conclusion}

This paper uses intraregional boundaries intersection group dynamic subdivision and consolidation to adapt the traffic signal control strategy. Then this paper optimizes intraregional boundaries intersection group traffic signal coordination strategy based on organic computing. This paper aims to solve the problem of traffic flow agglomeration effect characteristics and meet the rapid evacuation requirement in 
sudden disaster. From the simulation results, it is shown that only using intraregional boundaries traffic signal control and coordination can reduce the intraregional boundaries traffic flow delay and queue length but cannot significantly improve the traffic network performance: traffic network performance efficiency increases by less than $20 \%$ in congestion traffic flow status. When the system uses interregional traffic signal strategic coordination and intraregional boundaries traffic signal coordination together, it can obviously improve the traffic network performance, that is, traffic network performance efficiency promotes more than $30 \%$ in congestion traffic flow status, and can rapidly evacuate the traffic flow in disaster affected region.

\section{Conflict of Interests}

The authors declare that there is no conflict of interests regarding the publication of this paper.

\section{Acknowledgments}

The authors acknowledge the National Science Foundation of China (Grant no. 51308248), Specialized Research Fund for the Doctoral Program of Higher Education of China (Grant no. 20110061120043), National Science Foundation for Post-Doctoral Scientists of China (Grant no. 2013T60331), and Youth Scientific Research Fund of Jilin (Grant no. 20140520134JH) which partly supported this work.

\section{References}

[1] L. T. Lin and S. M. Tsao, "A system approach on signal grouping for area wide control of computerized traffic system," in Proceedings of the 79th Transportation Research Board Annual Meeting, p. 21, Transportation Research Board, Washington, DC, USA, 2001.

[2] J. E. Moore and P. P. Jovanis, "Statistical designation of traffic control subareas," Journal of Transportation Engineering, vol. 111, no. 3, pp. 208-223, 1985.

[3] S. C. Wong, "Group-based optimisation of signal timings using the transyt traffic model," Transportation Research B: Methodological, vol. 30, no. 3, pp. 217-244, 1996.

[4] H. Pranevicius and T. Kraujalis, "Knowledge based traffic signal control model for signalized intersection," Transport, vol. 27, no. 3, pp. 263-267, 2012.

[5] M. J. H. Chandler, "Organisation of traffic control in metropolitan areas," Traffic Engineering and Control, vol. 25, no. 12, pp. 599-610, 1984.

[6] L. Wang and Y. Bie, "An adaptive model for calculating the correlation degree of multiple adjacent signalized intersections," Mathematical Problems in Engineering, vol. 2013, Article ID 247184, 13 pages, 2013.

[7] Y. Hsu and S. Peeta, "Risk-based spatial zone determination problem for stage-based evacuation operations," Transportation Research C: Emerging Technologies, vol. 41, pp. 73-89, 2014.

[8] J. Lee, B. Abdulhai, A. Shalaby, and E.-H. Chung, "Real-time optimization for adaptive traffic signal control using genetic algorithms," Journal of Intelligent Transportation Systems: Technology, Planning, and Operations, vol. 9, no. 3, pp. 111-122, 2005.
[9] W. H. Kraft, Traffic Engineering Handbook, Insitute of Transportation Engineers, 6th edition, 2003.

[10] H. Qudrat-Ullah, Complex Decision Making-Theory and Practice, Springer, Berlin, Germany, 2008.

[11] C. Kahraman, Fuzzy Multi-Criteria Decision Making: Theory and Applications with Recent Developments, Springer, New York, NY, USA, 2008.

[12] H. Prothmann, Organic Traffic Control, KIT Scientific Publishing, Karlsruhe, Germany, 2011.

[13] S. Tomforde, H. Prothmann, F. Rochner et al., "Decentralised progressive signal systems for organic traffic control," in Proceedings of the 2nd IEEE International Conference on SelfAdaptive and Self-Organizing Systems (SASO '08), IEEE Computer Society, Venice, Italy, 2008.

[14] V. Dinopoulou, C. Diakaki, and M. Papageorgiou, "Applications of the urban traffic control strategy TUC," European Journal of Operational Research, vol. 175, no. 3, pp. 1652-1665, 2006.

[15] U. Ratsch, P. D. M. M. Richter, and P. D. I. Stamatescu, Intelligence and Artificial Intelligence, Springer, Berlin, Germany, 1998.

[16] E.-Y. Kim, S.-C. Kim, and B.-S. Seong, "Structure of attractive and repulsive hard-core Yukawa fluids: density functional perturbation theory," Fluid Phase Equilibria, vol. 308, no. 1-2, pp. 90-97, 2011.

[17] C.-Y. Lin, B.-W. Gong, D.-X. Zhao, and Z.-S. Yang, "Interregional traffic signal coordination control under sudden disaster based on game theory," Journal of Jilin University, vol. 41, no. 5, pp. 1257-1261, 2011.

[18] A. Jahangiri, S. Afandizadeh, and N. Kalantari, “The optimization of traffic signal timing for emergency evacuation using the simulated annealing algorithm," Transport, vol. 26, no. 2, pp. 133-140, 2011. 


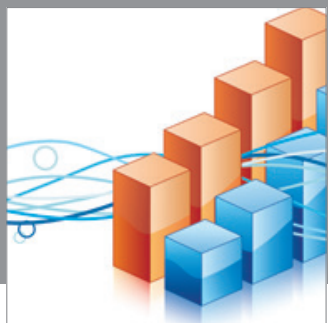

Advances in

Operations Research

mansans

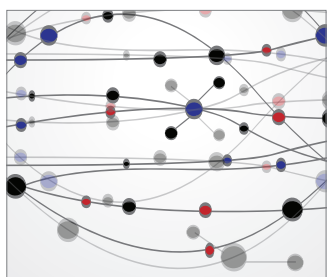

The Scientific World Journal
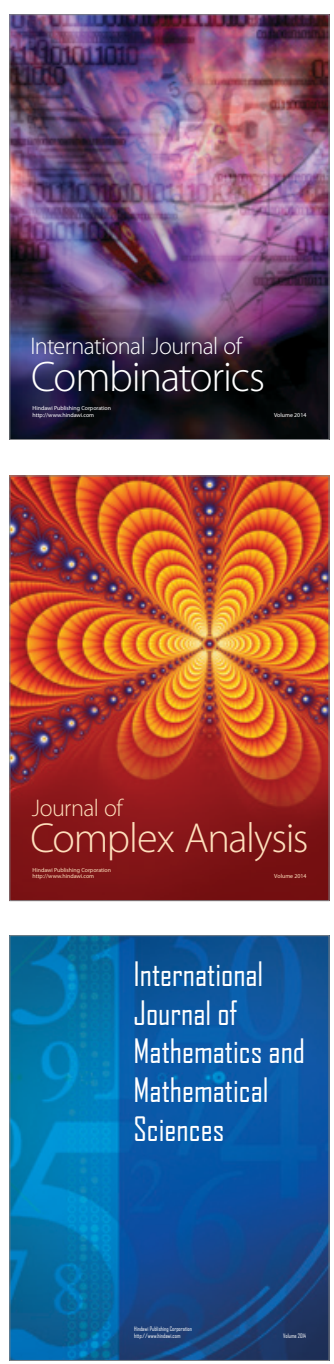
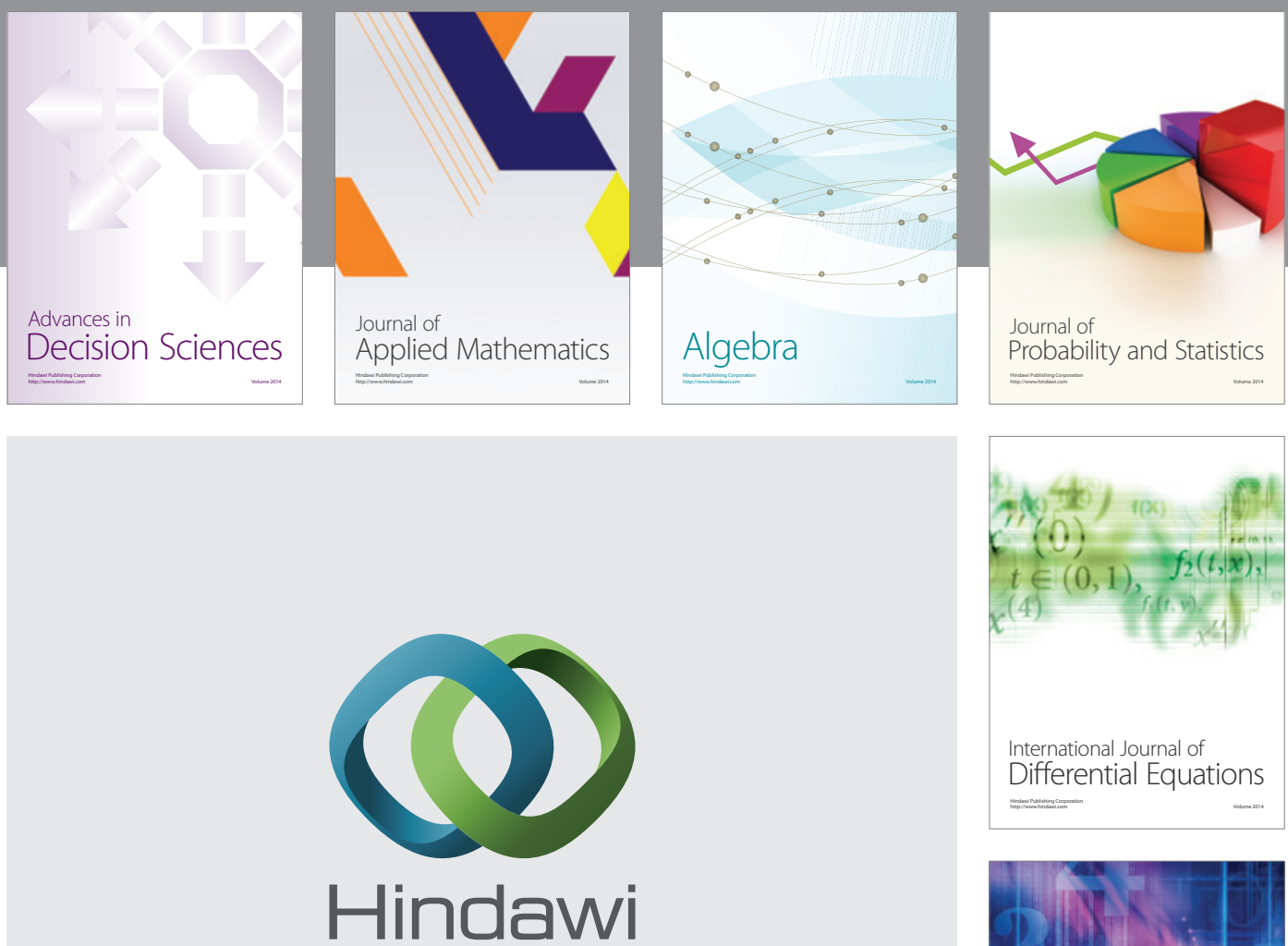

Submit your manuscripts at http://www.hindawi.com
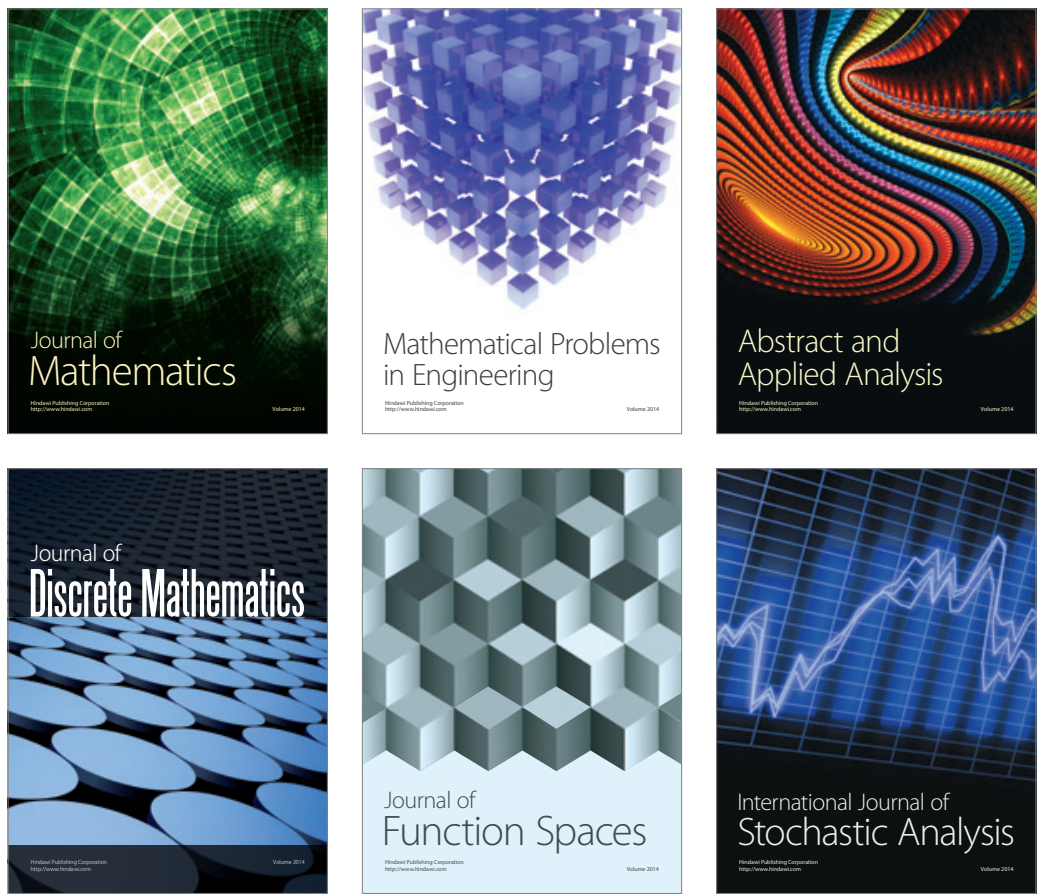

Journal of

Function Spaces

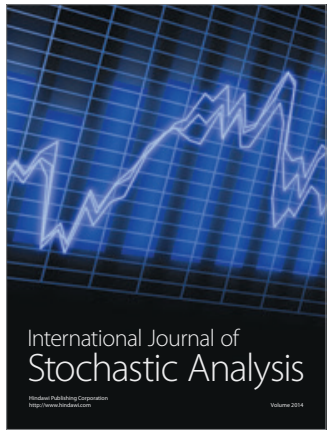

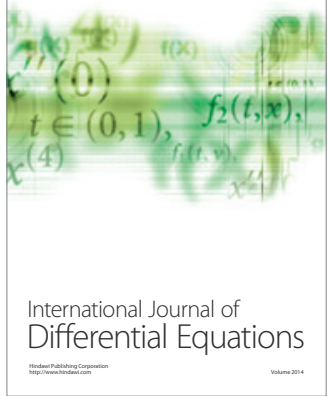
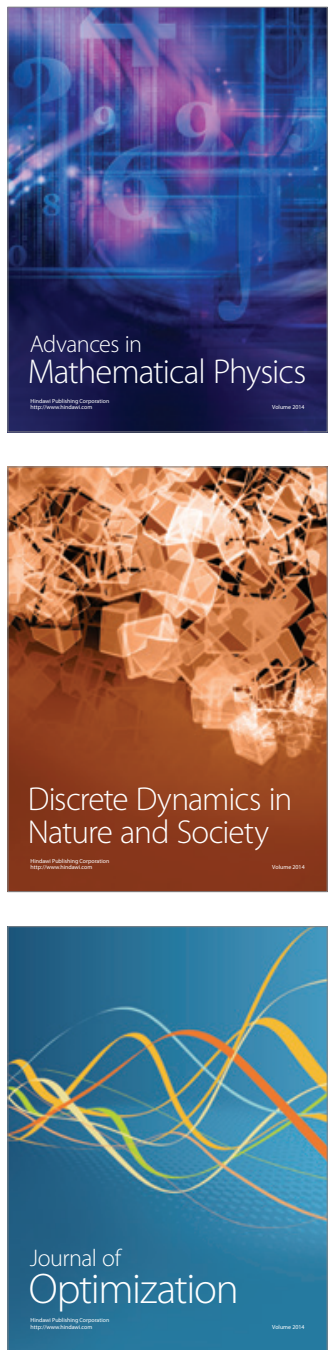